\title{
ORBITAL ANGULAR MOMENTUM
}

\section{HOW TO DEFINE IT AND HOW TO MEASURE IT}

\author{
Elliot Leader ${ }^{\text {a }}$ \\ ${ }^{1}$ Imperial College London
}

\begin{abstract}
In the context of the controversy concerning the ambiguities in the definition of quark and gluon angular momentum we explain pedagogically the origin of these ambiguities and stress that there are fundamentally only three physically relevant variants. We give precise expressions for the sum rules involving them. We consider their measurement, both experimentally and on the lattice, and discuss some attempts to calculate them in models.
\end{abstract}

\section{Introduction}

It has been emphasized for a long time that the angular momentum (AM) of a photon cannot be split, in a gauge invariant way, into a spin and orbital (OAM) part. The same is true for gluons, yet many experimental groups believe they are measuring the gluon spin! In 2008 Chen, Lu, Sun, Wang and Goldman [1] claimed, effectively, that all the QED textbooks were wrong, and that it was possible to split the photon angular momentum, in $a$ gauge-invariant way, into a spin part and an orbital part. This unleashed a deluge of papers, most of them very technical, and causing confusion by effectively implying that there are an infinite number of ways of defining the AM. In our recent Physics Report, with C. Lorcé [2], while covering all the technical developments, we conclude that there are only three physically relevant versions and indicate what parts of our review to read for the essential physical implications. This talk follows the latter, non-technical route, discussing ambiguities or variants in the definition of $\boldsymbol{L}$, the three fundamental versions, how to measure them experimentally, or on a lattice, and discussing some model calculations.

For simplicity, ambiguities, variants etc will all be illustrated mainly in QED. Note that throughout we consider a longitudinally polarized nucleon moving along $O Z$, and only discuss $L_{z}$.

\section{The physically meaningful versions of the angular momentum}

There are two kinds of variants: (1) there is a difference between canonical and kinetic angular momentum and (2) there is a difference, depending on the form of dynamics being used i.e. between instant form and light-front dynamics

The difference between canonical and kinetic has nothing to do with Field Theory. It is hidden in Undergraduate Physics! In Classical Dynamics the kinetic momentum is defined as mass times velocity

$$
\boldsymbol{p}_{\text {kin }}=m \boldsymbol{v}=m \dot{\boldsymbol{x}} .
$$

It follows the motion of the particle and the non-relativistic expression for the kinetic energy is

$$
E_{\mathrm{kin}}=\boldsymbol{p}_{\mathrm{kin}}^{2} / 2 m \text {. }
$$

In Quantum Mechanics one has the Heisenberg uncertainty relations between position and momentum

$$
\left[x_{i}, p_{j}\right]=i \hbar \delta_{i j} \text {. }
$$

This $\boldsymbol{p}$ is NOT the kinetic momentum. It is the canonical momentum, defined as

$$
\boldsymbol{p}=\partial L / \partial \dot{\boldsymbol{x}}
$$

where $L$ is the Lagrangian of the system.

Let's compare $\boldsymbol{p}_{\text {can }}$ with $\boldsymbol{p}_{\text {kin }}$. For a particle moving in a potential $V(\boldsymbol{x})$

$$
L=E_{\mathrm{kin}}-V=\frac{1}{2} m \dot{\boldsymbol{x}}^{2}-V(\boldsymbol{x})
$$

so that

$$
\boldsymbol{p}_{\mathrm{can}}=m \dot{\boldsymbol{x}}=\boldsymbol{p}_{\mathrm{kin}},
$$

and there is no distinction between kinetic and canonical momentum.

What happens if an electromagnetic field is present? Consider a classical problem: a charged particle, say an electron with charge $e$, moving in a fixed homogeneous external magnetic field $\boldsymbol{B}=(0,0, B)$. The particle follows a helical trajectory, so that at each instant, the particle kinetic momentum $\boldsymbol{p}_{\text {kin }}$ points toward a different direction. The Lagrangian is given by

$$
L=\frac{1}{2} m \dot{\boldsymbol{x}}^{2}-e \dot{\boldsymbol{x}} \cdot \boldsymbol{A}
$$


where $\boldsymbol{A}$ is the vector potential responsible for the magnetic field $\boldsymbol{B}=\boldsymbol{\nabla} \times \boldsymbol{A}$. It leads to

$$
\boldsymbol{p}_{\mathrm{can}}=\boldsymbol{p}_{\mathrm{kin}}[\boldsymbol{x}(t)]-e \boldsymbol{A}[\boldsymbol{x}(t)]
$$

Under a gauge transformation $\boldsymbol{A}$ changes, but that does not affect the physical motion of the particle. But, it clearly changes $\boldsymbol{p}_{\text {can }}$. $\boldsymbol{p}_{\text {can }}$ is a gauge non-invariant quantity, and a key issue in the recent controversy is whether such a quantity is measurable? The situation for angular momentum is quite analogous.

Turning now to the present angular momentum controversy, consider the QCD sum rule relating the spin of the nucleon to the angular momentum of its constituents

$$
\frac{1}{2}=\left\langle\left\langle S_{z}^{q}\right\rangle\right\rangle+\left\langle\left\langle L_{z}^{q}\right\rangle\right\rangle+\left\langle\left\langle S_{z}^{G}\right\rangle\right\rangle+\left\langle\left\langle L_{z}^{G}\right\rangle\right\rangle
$$

It is totally intuitive; can't be incorrect. But the operators $L^{q, G}$ and $S^{G}$ are not gauge invariant. It is based on the QCD canonical version of the angular momentum $\boldsymbol{J}$. In QED $q \rightarrow$ electron, $G \rightarrow$ photon

$$
\begin{aligned}
\boldsymbol{J}_{\mathrm{can}} & =\underbrace{\int d^{3} x \psi^{\dagger} \frac{1}{2} \boldsymbol{\Sigma} \psi}_{\boldsymbol{S}_{\mathrm{can}}^{e}}+\underbrace{\int d^{3} x \psi^{\dagger}\left(\boldsymbol{x} \times \frac{1}{i} \boldsymbol{\nabla}\right) \psi}_{\boldsymbol{L}_{\mathrm{can}}^{e}} \\
+ & \underbrace{\int d^{3} x \boldsymbol{E} \times \boldsymbol{A}}_{\boldsymbol{S}_{\mathrm{can}}^{\gamma}}+\underbrace{\int d^{3} x E^{i}\left(\boldsymbol{x} \times \boldsymbol{\nabla} A^{i}\right)}_{\boldsymbol{L}_{\mathrm{can}}^{\gamma}}
\end{aligned}
$$

This is nice, because it splits $\boldsymbol{J}^{\gamma, G}$ into $\boldsymbol{S}^{\gamma, G}+\boldsymbol{L}^{\gamma, G}$ and we claim to measure the gluon spin.

Usually we write Eq. (9) in the Jaffe-Manohar (JM) form:

$$
\frac{1}{2}=\frac{1}{2} a_{0}+\Delta G+\left\langle\left\langle L_{z}^{q}\right\rangle\right\rangle+\left\langle\left\langle L_{z}^{G}\right\rangle\right\rangle
$$

where

$$
a_{0}=\text { axial charge of nucleon, }
$$

but we should write the Jaffe-Manohar sum rule in the more precise the form :

$$
\frac{1}{2}=\frac{1}{2} a_{0}+\Delta G+\left\langle\left\langle L_{c a n, z}^{q}\right\rangle\right\rangle+\left\langle\left\langle L_{c a n, z}^{G}\right\rangle\right\rangle .
$$

But this is still not completely accurate: Danger! $\Delta G$ is a gauge invariant quantity but $\left\langle\left\langle S_{\text {can }, z}^{G}\right\rangle\right\rangle$ is (supposedly) not. However one can show that as the nucleon momentum $P \rightarrow \infty$

$$
\Delta G=\left.\left\langle\left\langle S_{\text {can }, z}^{G}\right\rangle\right\rangle\right|_{\text {Gauge } A^{+}=0} .
$$

Thus it is $\left.\left\langle\left\langle L_{\mathrm{can}, z}^{q}\right\rangle\right\rangle\right|_{A^{+}=0}$ that should appear in the JM sum rule.

There is another subtlety. $\boldsymbol{J}_{\text {can }}$ was defined in terms of the generalised angular momentum density tensor $M^{\mu \nu \rho}(t, \boldsymbol{x})$ as

$$
J_{\text {can }}^{i}=\frac{1}{2} \epsilon^{i j k} \int d^{3} x M^{0 j k}(t, x)
$$

This is the instant form: an integral over space at fixed time of $M^{0 j k}(t, \boldsymbol{x})$

In light-front dynamics, the role of time is played by $x^{+}$ and the integral is over $d x^{-} d^{2} \boldsymbol{x}_{\perp}$ of $M^{+j k}\left(x^{+}, x^{-}, \boldsymbol{x}_{\perp}\right)$. So there is $\boldsymbol{J}_{\text {can }}^{\text {inst }}$ and $\boldsymbol{J}_{\text {can }}^{\text {If }}$ and, analogously, $\boldsymbol{L}_{\text {can }}^{q, i n s t}$ and $\boldsymbol{L}_{\text {can }}^{q, \text { If }}$ with, as can be shown,

$$
\lim _{P \rightarrow \infty}\left\langle\left\langle\boldsymbol{L}_{\mathrm{can}}^{q, \text { inst }}\right\rangle\right\rangle=\left\langle\left\langle\boldsymbol{L}_{\mathrm{can}}^{q, l f}\right\rangle\right\rangle .
$$

The kinetic version, called Belinfante in Field Theory, is

$$
\begin{aligned}
\boldsymbol{J}_{\mathrm{Bel}}= & \underbrace{\int \mathrm{d}^{3} x \bar{\psi}\left[\boldsymbol{x} \times \frac{1}{2}\left(\gamma^{0} i \boldsymbol{D}+\boldsymbol{\gamma} i D^{0}\right)\right] \psi}_{\boldsymbol{J}_{\mathrm{Bel}}^{e}} \\
& +\underbrace{\int \mathrm{d}^{3} x \boldsymbol{x} \times(\boldsymbol{E} \times \boldsymbol{B})}_{\boldsymbol{J}_{\mathrm{Bel}}^{\gamma}}
\end{aligned}
$$

where the covariant derivative is given by $\boldsymbol{D}=\boldsymbol{\partial}+i e \boldsymbol{A} \equiv$ $-\boldsymbol{\nabla}+i e \boldsymbol{A}$ and $D^{0}=\partial_{t}+i e A^{0}$. Notice that there is no electron spin, no photon spin. But each term is gauge invariant.

Using the Equations of Motion and discarding a surface term at infinity, coming from integrating a divergence term of the form $\boldsymbol{\nabla} \cdot$ term , yields the form used by $\mathrm{Ji}$ :

$$
\begin{aligned}
\boldsymbol{J}_{\mathrm{Ji}}= & \underbrace{\int \mathrm{d}^{3} x \psi^{\dagger} \frac{1}{2} \boldsymbol{\Sigma} \psi}_{\boldsymbol{S}_{\mathrm{Ji}}^{e}}+\underbrace{\int \mathrm{d}^{3} x \psi^{\dagger}(\boldsymbol{x} \times i \boldsymbol{D}) \psi}_{\boldsymbol{L}_{\mathrm{Ji}}^{e}} \\
& +\underbrace{\int \mathrm{d}^{3} x \boldsymbol{x} \times(\boldsymbol{E} \times \boldsymbol{B})}_{\boldsymbol{J}_{\mathrm{Ji}}^{\gamma}} .
\end{aligned}
$$

All pieces are gauge invariant, but $\boldsymbol{J}_{\mathrm{Ji}}^{\gamma}$ is not split into spin and orbital parts.

These are the instant form expressions. As with the canonical case one can define light-front forms, but in this case, in QCD,

$$
\left\langle\left\langle\boldsymbol{L}_{\mathrm{Ji}}^{q, i n s t}\right\rangle\right\rangle=\left\langle\left\langle\boldsymbol{L}_{\mathrm{Ji}}^{q, l f}\right\rangle\right\rangle
$$

In summary there exist THREE different OAM expectation values of interest

$$
\left.\left.\left\langle\left\langle L_{\mathrm{can}, z}^{\mathrm{inst}, q}\right\rangle\right\rangle\right|_{A^{+}=0} \quad\left\langle\left\langle L_{\mathrm{can}, z}^{\mathrm{lf}, q}\right\rangle\right\rangle\right|_{A^{+}=0} \quad\left\langle\left\langle L_{\mathrm{Ji}, z}^{q}\right\rangle\right\rangle
$$

and, don't forget, they are renormalization scale dependent.

\section{Measurement and models of the orbital angular momentum}

3.1 The kinetic version : $\left\langle\left\langle L_{\mathbf{J i}, z}^{q}\right\rangle\right\rangle$

(1) The Ji relation [3] relates $\left\langle\left\langle J_{\mathrm{Ji}, z}^{q}\right\rangle\right\rangle$ to Generalized Parton Distributions (GPDs $H$ and $E$ )

$$
\left\langle\left\langle J_{\mathrm{Ji}, z}^{q}\right\rangle\right\rangle=\frac{1}{2} \int_{-1}^{1} \mathrm{~d} x x\left[H_{q}(x, 0,0)+E_{q}(x, 0,0)\right]
$$

Thus

$$
\left\langle\left\langle L_{\mathrm{Ji}, z}^{q}\right\rangle\right\rangle=\frac{1}{2} \int_{-1}^{1} \mathrm{~d} x x\left[H_{q}(x, 0,0)+E_{q}(x, 0,0)\right]-\frac{1}{2} a_{0}^{q}
$$


where $a_{0}^{q}$ is the contribution to $a_{0}$ (or $g_{A}^{(0)}$ ), the flavorsinglet axial charge of the nucleon, from a quark plus antiquark of given flavor To utilize this we have input data from two sources:

(1.i) Extraction of $H$ from data on quark PDFs and $E$ from DVCS data, EM Form Factors etc. This is not easy and requires much more data. A recent study by Diehl and Kroll [4] concentrates on the connection with electromagnetic form factors and thus only provides information about the "valence" GPDs e.g. $E_{u}-E_{\bar{u}}$. With $J_{q} \equiv\left\langle\left\langle J_{\mathrm{Ji}, z}^{q}\right\rangle\right\rangle$ they find

$$
J_{u}^{v a l}=0.230_{-0.024}^{+0.009} \quad J_{d}^{v a l}=-0.004_{-0.016}^{+0.010} .
$$

On the other hand Gonzalez-Hernandez, Liuti, Goldstein and Katuria [5] obtain values

$$
J_{u}^{v a l}=0.286 \pm 0.011 \quad J_{d}^{v a l}=-0.049 \pm 0.007
$$

which disagree significantly with Diehl and Kroll. Clearly the subject is still in its early stages and much more accurate data will surely emerge soon.

(1.ii) Lattice calculations. The latest results are from Deka et al [6]

The calculation uses the quenched approximation (no quark-antiquark loops) and includes both connected insertions (CI) (current connects only to valence quark lines) and disconnected insertions (DI) (current also connects to quark loops, but not dynamical). The DI contributions turn out to be large. Figs. 1 and 2 show the beautiful Deka et al results for

$$
L_{q} \equiv\left\langle\left\langle L_{\mathrm{Ji}, z}^{q}\right\rangle\right\rangle \quad J_{q} \equiv\left\langle\left\langle J_{\mathrm{Ji}, z}^{q}\right\rangle\right\rangle
$$

where, recall, $q$ means quark plus antiquark contribution.

Their values for the total AM are

$$
J_{u}=0.369 \pm 0.059 \quad J_{d}=-0.018 \pm 0.042 .
$$

The Table shows the various contributions to the orbital angular momentum:

$$
\begin{array}{cccc} 
& C I & D I & \text { Total } \\
\cline { 3 - 4 } L_{u} & -0.11 \pm 0.08 & 0.08 \pm 0.005 & -0.025 \pm 0.080 \\
L_{d} & 0.11 \pm 0.08 & 0.08 \pm 0.005 & 0.19 \pm 0.07
\end{array}
$$$$
\text { NB } L_{u}-L_{d} \quad \text { is negative } \quad=-0.22 \pm 0.11
$$

This will be a key issue when we come to discuss models. If we make the reasonable, but non-rigorous assertion that for the DI contributions, $D I(u)=D I(\bar{u}), D I(d)=D I(\bar{d})$ then we obtain for the valence contributions

$$
J_{v a l}^{u}=0.335 \quad J_{v a l}^{d}=-0.052
$$

which are not too far from the values of GonzalezHernandez, Liuti, Goldstein and Katuria [5] given above.
2) The relation to twist-3 GPD $G_{2}^{q}$ of Kiptily and Polyakov [7]. One finds

$$
\left\langle\left\langle L_{\mathrm{Ji}, z}^{q}\right\rangle\right\rangle=-\int_{-1}^{1} \mathrm{~d} x \times G_{2}^{q}(x, 0,0),
$$

a relation which was first obtained by Penttinen, Polyakov, Shuvaev and Strikman in the parton model [8] and later confirmed in QCD by Hatta and Yoshida [9]. To the best of my knowledge this has not yet been used because it is extremely difficult to extract information on such a twist-3 GPD from experiment.

3) The Lorcé and Pasquini [10] relation to Generalized Transverse Momentum Distributions (GTMDs)

$$
\left\langle\left\langle L_{\mathrm{Ji}, z}^{q}\right\rangle\right\rangle=-\int \mathrm{d} x \mathrm{~d}^{2} k_{\perp} \frac{\boldsymbol{k}_{\perp}^{2}}{M^{2}} F_{1,4}^{q}\left(x, \boldsymbol{k}_{\perp}, \Delta=0 ; \boldsymbol{W}_{\text {straight }}\right),
$$

where the Wilson line $\mathcal{W}_{\text {straight }}$ connects the points $-\frac{z}{2}$ and $\frac{z}{2}$ by a direct straight line. At present there is no clear way of extracting the twist-2 GTMDs from experimental data, but they can be calculated in models.

\subsection{The canonical version : $\left\langle\left\langle L_{\text {can }, z}^{q}\right\rangle\right\rangle$}

Lorcé, Pasquini [10] have shown that the canonical version can also be obtained from a twist-2 GTMD, but defined with a different Wilson line

$\left.\left\langle\left\langle L_{\mathrm{can}, z}^{l f, q}\right\rangle\right\rangle\right|_{A^{+}=0}=-\int \mathrm{d} x \mathrm{~d}^{2} k_{\perp} \frac{\boldsymbol{k}_{\perp}^{2}}{M^{2}} F_{1,4}^{q}\left(x, \boldsymbol{k}_{\perp}, \Delta=0 ; \mathcal{W}_{\mathrm{LF}}\right)$,

where the staple-like Wilson line $\mathcal{W}_{\mathrm{LF}}$ connects the points $-\frac{z}{2}$ and $\frac{z}{2}$ via the intermediary points $-\frac{z}{2} \pm \infty^{-}$and $\frac{z}{2} \pm$ $\infty^{-}$by straight lines. At present it is not clear how one could obtain this GTMD from experiment. It is interesting that in changing the shape of the Wilson line one obtains either the kinetic or the canonical quark orbital angular momentum.

\section{Model calculations}

There are four types of QCD models: none have genuine gluon degrees of freedom

- Light-Front Constituent Quark Model (LFCQM)

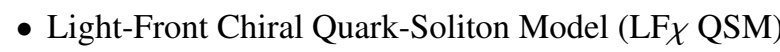

- MIT Bag Model

- Myher-Thomas Cloudy Bag Model with OGE

\subsection{The sign of $L_{u}-L_{d}$}

As will be seen shortly, all models, with the exception of the LF $\chi$ QSM lead to positive values of $L_{u}-L_{d}$.

However, a key question is: at what scale is the model valid?

Presumably it should be a very low scale, if there are no gluon degrees of freedom. The scale is usually fixed by 


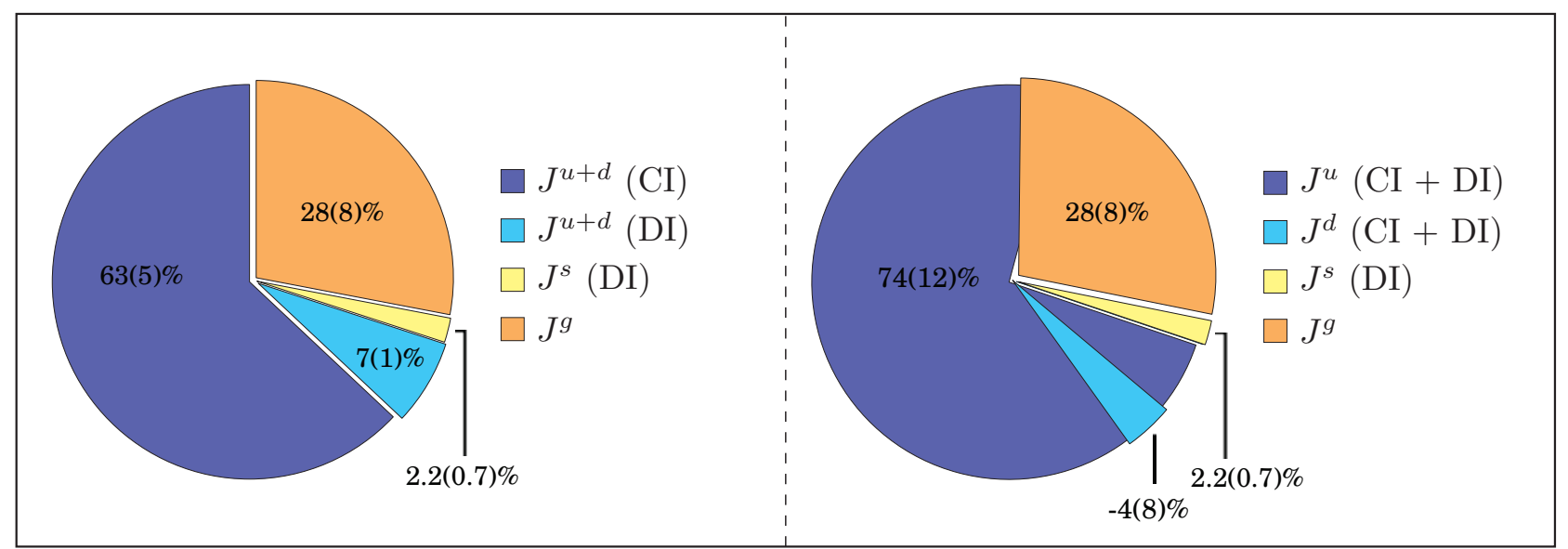

Figure 1. Flavour dependence of the total angular momentum. Courtesy of Keh-Fei Liu

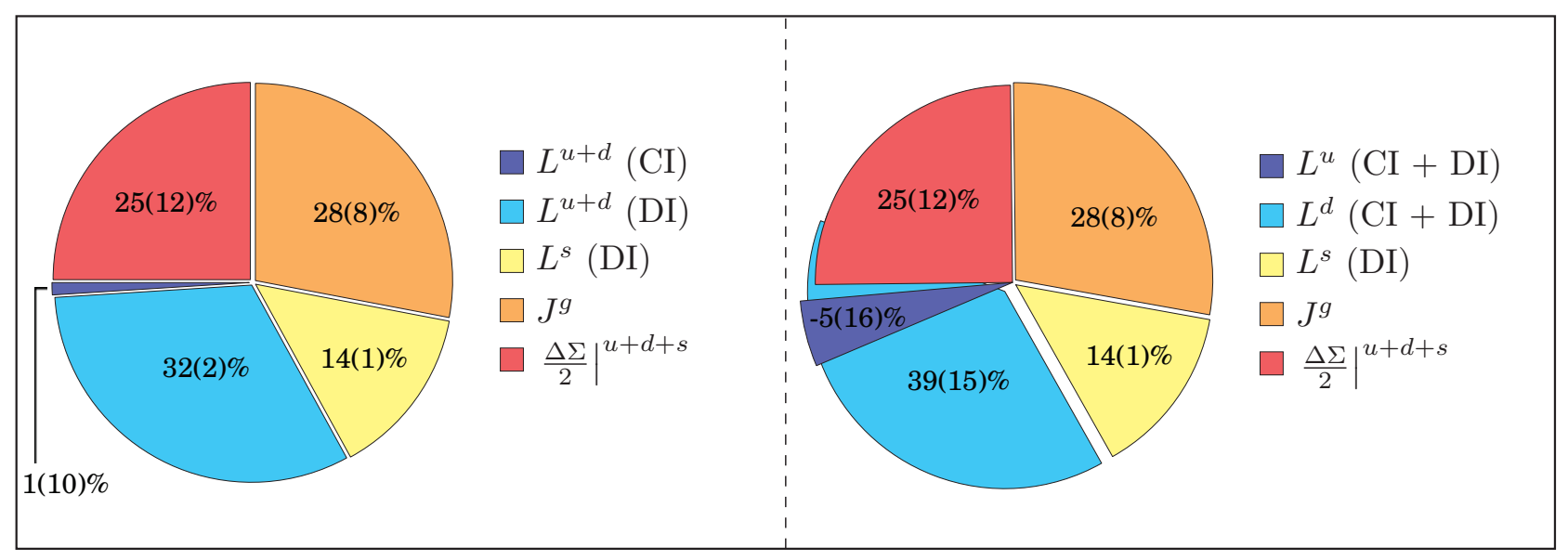

Figure 2. Flavour dependence of the orbital angular momentum. Courtesy of Keh-Fei Liu

forcing the model to agree with ONE measured observable. Typically this leads to a scale of $0.16-0.36 \mathrm{GeV}^{2}$. Thomas [11] suggests that the positive values of $L_{u}-L_{d}$ at the low scale of the models is not a problem since there is a cross-over in $L_{u}-L_{d}$ due to evolution. This is shown for the Myher-Thomas model in Fig. 3, where I have inserted the values found by Deka et al; the agreement is very good. A similar evolution, starting with the correct sign $L_{u}-L_{d}<0$ from the LF $\chi$ QSM at a low scale, as used by Wakamatsu [12], gives poor agreement with the Lattice results after evolution. The problem is: can evolution be trusted at such low scales where $\alpha_{s}$ is not small???

\subsection{Kinetic vs Canonical in models}

Recall that

$$
\boldsymbol{L}_{J i}^{q}=\left\langle\left\langle\int \mathrm{d}^{3} x \psi^{\dagger}(\boldsymbol{x} \times i \boldsymbol{D}) \psi\right\rangle\right\rangle
$$

and

$$
\boldsymbol{L}_{c a n}^{q}=\left\langle\left\langle\int d^{3} x \psi^{\dagger}\left(\boldsymbol{x} \times \frac{1}{i} \boldsymbol{\nabla}\right) \psi\right\rangle\right\rangle .
$$

Since models usually have no gluon degrees of freedom, $\boldsymbol{D}=-\boldsymbol{\nabla}$, so we expect that

$$
\boldsymbol{L}_{J i}^{q}=\boldsymbol{L}_{c a n}^{q}
$$

What do the models calculate?

(i) Expanding the nucleon state in terms of light-front wave functions in the definition of $\boldsymbol{L}_{c a n}^{q}$ restricted to the 3-quark sector,

$$
\begin{aligned}
& \left.\ell_{\mathrm{can}, z}^{q} \equiv\left\langle\left\langle L_{\mathrm{can}, z}^{l f, q}\right\rangle\right\rangle\right|^{\text {model }} \\
= & \sum_{\{\lambda\}} \int[\mathrm{d} x]_{3}\left[\mathrm{~d}^{2} k_{\perp}\right]_{3} \Psi_{3}^{*+}\left(\left\{x, \boldsymbol{k}_{\perp}, \lambda\right\}\right) \\
\times & \sum_{l, r(q)}\left(\delta_{r l}-x_{l}\right)\left(\boldsymbol{k}_{r \perp} \times \frac{1}{i} \nabla_{\boldsymbol{k}_{\perp \perp}}\right)_{z} \Psi_{3}^{+}\left(\left\{x, \boldsymbol{k}_{\perp}, \lambda\right\}\right)
\end{aligned}
$$

The peculiar structure

$$
\sum_{l, r(q)}\left(\delta_{r l}-x_{l}\right)\left(\boldsymbol{k}_{r \perp} \times \frac{1}{i} \boldsymbol{\nabla}_{\boldsymbol{k}_{l \perp}}\right)
$$

in Eq. (34) requires some explanation. This is the intrinsic OAM defined with respect to the transverse center of momentum . Non-relativistically the centre of mass is defined 


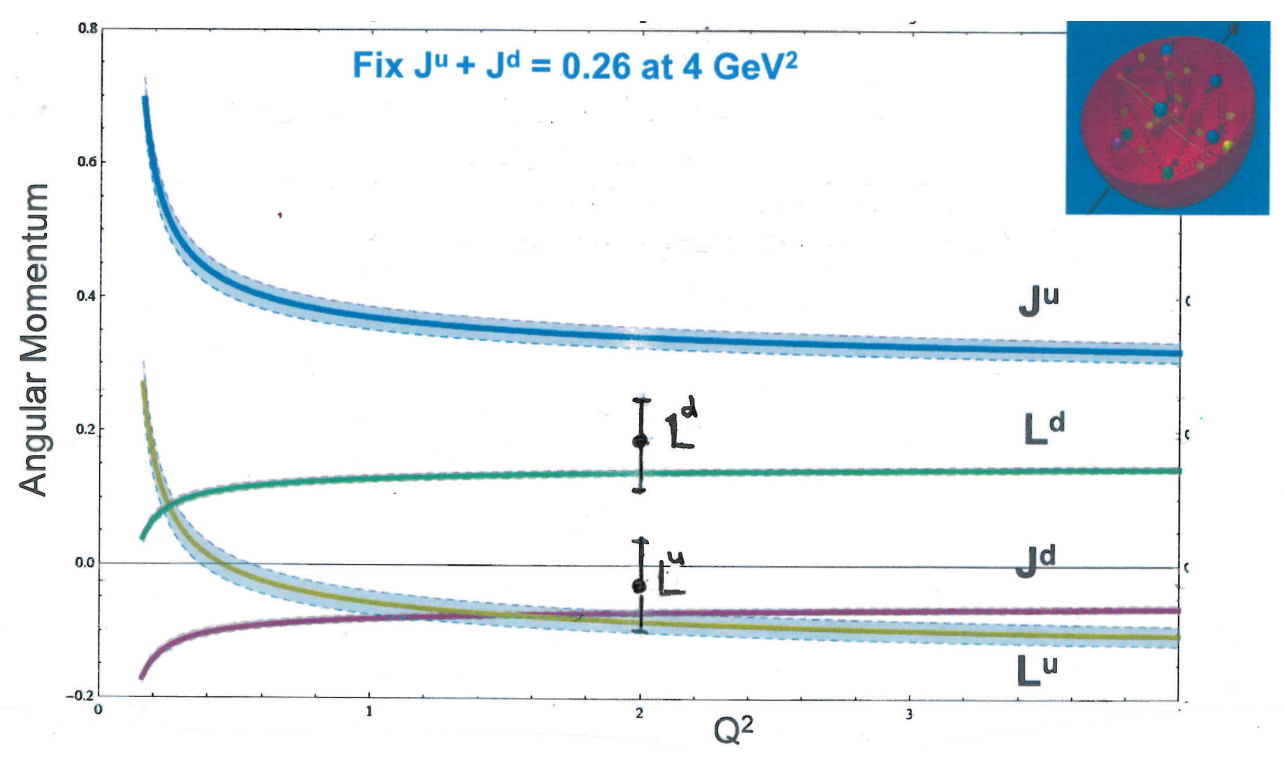

Figure 3. Evolution of $L_{u}-L_{d}$ in the Myher-Thomas model. Courtesy of Tony Thomas. I have inserted the results of Deka et al [6]

by: $\boldsymbol{R}_{C M}=\sum_{l}\left(\frac{m_{l}}{M}\right) \boldsymbol{r}_{l}$. In relativity the centre of inertia or centre of momentum is is given by

$$
\boldsymbol{R}=\sum_{l}\left(\frac{E_{l}}{E}\right) \boldsymbol{r}_{l} \stackrel{\text { fast quark }}{\longrightarrow} \sum_{l} x_{l} \boldsymbol{r}_{l}
$$

The structure referred to is designed to yield the angular momentum about the transverse version of this introduced by Burkardt [13]

$$
\boldsymbol{R}_{\perp}=\sum_{l=1}^{3} x_{l} \boldsymbol{r}_{l \perp}
$$

Thus one should define the $Z$-component of intrinsic OAM for a quark $q$ using $-\left(\boldsymbol{k}_{q \perp} \times \boldsymbol{b}_{q}\right)$ where $\boldsymbol{b}_{q}$ is the impact parameter

$$
\begin{gathered}
\boldsymbol{b}_{q}=\boldsymbol{r}_{q, \perp}-\boldsymbol{R}_{\perp}=\boldsymbol{r}_{q, \perp}-\sum_{l=1}^{3} x_{l} \boldsymbol{r}_{l \perp} \\
=\left(1-x_{q}\right) \boldsymbol{r}_{q, \perp}-\sum_{l \neq q} x_{l} \boldsymbol{r}_{l \perp} .
\end{gathered}
$$

In the momentum representation $\frac{1}{i} \nabla_{\boldsymbol{k}_{l}} \rightarrow \boldsymbol{r}_{l}$. Thus

$$
\sum_{l}\left(\delta_{q l}-x_{l}\right)\left(\boldsymbol{k}_{q \perp} \times \frac{1}{i} \nabla_{\boldsymbol{k}_{\perp \perp}}\right) \rightarrow\left(\boldsymbol{k}_{q \perp} \times \boldsymbol{b}_{q}\right)
$$

(ii) One obtains the same result using a model of the GTMD in Eq. (30)

$$
\ell_{\mathrm{can}, z}^{q}=-\left.\int \mathrm{d} x \mathrm{~d}^{2} k_{\perp} \frac{\boldsymbol{k}_{\perp}^{2}}{M^{2}} F_{1,4}^{q}\left(x, \boldsymbol{k}_{\perp}, \Delta=0 ; \boldsymbol{W}_{\mathrm{LF}}\right)\right|^{\text {model }}
$$

(iii) For the kinetic version of the angular momentum, using a model of the GPDs in the Ji relation Eq. (21) one has

$$
\left.\ell_{\mathrm{kin}, z}^{q} \equiv\left\langle\left\langle L_{\mathrm{Ji}, z}^{q}\right\rangle\right\rangle\right|^{\text {model }}=\int_{-1}^{1} \mathrm{~d} x \ell_{\mathrm{kin}, z}^{q}(x)
$$

where

$$
\ell_{\mathrm{kin}, z}^{q}(x)=\left.\frac{1}{2}\left\{x\left[H_{q}(x, 0,0)+E_{q}(x, 0,0)\right]-2 S_{z}^{q}(x)\right\}\right|^{\text {model }}
$$

(iv) There exists also a so-called "naive" version of $\left\langle\left\langle L_{\text {can }, z}^{l f, q}\right\rangle\right\rangle$ which has been evaluated using light-front wave functions

$$
\begin{aligned}
& \left.\mathcal{L}_{\text {can }, z}^{q} \equiv\left\langle\left\langle L_{\text {can }, z}^{I f, q}\right\rangle\right\rangle\right|^{\text {naive, model }} \\
& =\sum_{\{\lambda\}} \int[\mathrm{d} x]_{3}\left[\mathrm{~d}^{2} k_{\perp}\right]_{3} \Psi_{3}^{*+}\left(\left\{x, \boldsymbol{k}_{\perp}, \lambda\right\}\right) \\
& \quad \times \sum_{r(q)}\left(\boldsymbol{k}_{r \perp} \times \frac{1}{i} \nabla_{\boldsymbol{k}_{r \perp}}\right)_{z} \Psi_{3}^{+}\left(\left\{x, \boldsymbol{k}_{\perp}, \lambda\right\}\right)
\end{aligned}
$$

Here the factor $\left(\delta_{r l}-x_{l}\right)$ in Eq. (34 is replaced by $\delta_{r l}$. Therefore this is the angular momentum about the origin.

(v) In some models it appears that naive $\mathcal{L}_{\text {can, } z}^{q}$ can be obtained from the Pretzelosity. She et al [14] and Avakian et al [15] find

$$
\mathcal{L}_{\mathrm{can}, z}^{q}=-\int \mathrm{d} x \mathrm{~d}^{2} k_{\perp} \frac{\boldsymbol{k}_{\perp}^{2}}{2 M^{2}} h_{1 T}^{\perp q}\left(x, \boldsymbol{k}_{\perp}^{2}\right)
$$

but Lorce and Pasquini [16] have shown that this is only valid in a restricted class of models. It requires the instantform wave function $\psi(\{\boldsymbol{k}, \sigma\})$ to be a pure $s$-wave and related to the light-front wave function $\Psi\left(\left\{x, \boldsymbol{k}_{\perp}, \lambda\right\}\right)$ by just a Wigner rotation.

\subsection{Some model results}

In the table are shown results from the LFCQM and the LF $\chi$ QSM [10] 


\begin{tabular}{c|ccc|ccc}
\hline Model & \multicolumn{3}{|c|}{ LFCQM } & \multicolumn{3}{c}{ LF $\chi$ QSM } \\
$q$ & $u$ & $d$ & Total & $u$ & $d$ & Total \\
\hline$\ell_{\mathrm{kin}, z}^{q}$ & 0.071 & 0.055 & 0.126 & -0.008 & 0.077 & 0.069 \\
$\ell_{\mathrm{can}, z}^{q}$ & 0.131 & -0.005 & 0.126 & 0.073 & -0.004 & 0.069 \\
$\mathcal{L}_{\mathrm{can}, z}^{q}$ & 0.169 & -0.042 & 0.126 & 0.093 & -0.023 & 0.069 \\
\hline
\end{tabular}

- In each model the contributions of the $u$ quark to the three versions $\ell_{\mathrm{kin}, z}, \ell_{\mathrm{can}, z}, \mathcal{L}_{\mathrm{can}, z}$ are different, and similarly for the $d$ quark

- But in each model the total contribution of $(u+d)$ to $\ell_{\text {kin }, z}, \ell_{\text {can }, z}, \mathcal{L}_{\text {can }, z}$ is the same

- It is not surprising that $\ell_{\text {can }, z}^{q} \neq \mathcal{L}_{\text {can, },}^{q}$, and, as can be seen from comparing Eqs. $(34,43)$ one should expect to have the result found, i.e. that $\sum_{q} \ell_{\text {can }, z}^{q}=\sum_{q} \mathcal{L}_{\text {can, } z}^{q}$

- It is puzzling, given that there is no $A^{\mu}$ in the models, that $\ell_{\mathrm{kin}, z}^{q} \neq \ell_{\mathrm{can}, z}^{q}$. Probably this is because the Ji relation for $\ell_{\text {kin,z }}^{q}$ uses the QCD energy-momentum tensor, which is different from the one in the models

- In that case it may seem surprising that $\sum_{q} \ell_{\mathrm{kin}, z}^{q}=$ $\sum_{q} \ell_{\text {can }, z}^{q}$. Burkardt, at this Conference, has suggested that this occurs because all models are normalized so that

$$
\sum_{q} J_{\text {kin }, z}^{q}=\sum_{q} J_{\text {can }, z}^{q}=1 / 2
$$

and since the spin term $\sum_{q} S_{z}^{q}$ in each model doesn't depend on the label "kin" or "can" one will have

$$
\sum_{q} \ell_{\mathrm{kin}, z}^{q}=1 / 2-\sum_{q} S_{z}^{q}=\sum_{q} \ell_{\mathrm{can}, z}^{q} .
$$

There is a further puzzle. Burkardt and Hikmat [17] calculated $\ell_{\mathrm{kin}, z}^{q}$ via the Ji relation and $\ell_{\mathrm{can}, z}^{q}$ directly from the wave functions in the scalar diquark model, which has no $A^{\mu}$, and they obtained, as was expected, $\ell_{\mathrm{kin}, z}^{q}=\ell_{\mathrm{can}, z}^{q}$ But for the density in Bjorken-x they found, contrary to expectations, that

$$
\ell_{\mathrm{kin}, z}^{q}(x) \neq \ell_{\mathrm{can}, z}^{q}(x) .
$$

This implies that the Ji relation does not hold for the densities in $\mathrm{X}$-space i.e.

$$
\left\langle\left\langle J_{\mathrm{Ji}, z}^{q}(x)\right\rangle\right\rangle \neq \frac{1}{2} x\left[H_{q}(x, 0,0)+E_{q}(x, 0,0)\right]
$$

contrary to claim of Hoodbhoy, Ji and Lu [18]. The results in the latter paper depend on discarding an infinite number of surface terms, each arising from integrating a divergence term, and this is almost certainly unjustified.

\section{5 conclusions}

- There exist many other versions of the angular momentum, the so called, Gauge Invariant Extensions. They are theoretically interesting, but I believe they contain no new physics.
- There are just three physically relevant versions of the OAM:

$$
\left.\left.\left\langle\left\langle L_{\mathrm{can}, z}^{\text {inst }, q}\right\rangle\right\rangle\right|_{A^{+}=0} \quad\left\langle\left\langle L_{\mathrm{can}, z}^{l f, q}\right\rangle\right\rangle\right|_{A^{+}=0} \quad\left\langle\left\langle L_{\mathrm{Ji}, z}^{q}\right\rangle\right\rangle
$$

- All these can be related, in principle, to measurable quantities like GPDs and GTMDs, but measuring them from experiment is difficult and is a challenge for the future.

- $\left\langle\left\langle L_{\mathrm{Ji}, z}^{q}\right\rangle\right\rangle \equiv L_{q}$ can be calculated on a Lattice. Beautiful results have been obtained, in particular by Deka et al, who for the first time include the Disconnected Insertions, and find them to be very important. At $2 \mathrm{GeV}$, in the $\overline{M S}$ scheme, they find $L_{u}-L_{d}<0$.

- $\left\langle\left\langle L_{\text {can }, z}^{q}\right\rangle\right\rangle$ can be calculated in models where it is called $\ell_{\mathrm{can}, z}^{q}$. It is important to distinguish about what point the OAM is defined: naive about the origin or intrinsic about the transverse centre of momentum.

- $\left\langle\left\langle L_{\mathrm{Ji}, z}^{q}\right\rangle\right\rangle$ can be calculated in models, where it is called $\ell_{\mathrm{kin}, z}^{q}$. Almost all give $\ell_{\mathrm{kin}, z}^{u}-\ell_{\mathrm{kin}, z}^{d}>0$ at the scale where the model is supposed to be reliable. This is presumably a very low scale so that under evolution to the scale $2 \mathrm{GeV}$ this result may become compatible with the opposite sign as found in the Lattice calculations.

- In a model with no $A^{\mu}$ it is found, as expected, that $\ell_{\mathrm{kin}, z}^{q}=\ell_{\mathrm{can}, z}^{q}$, but, surprisingly that $\ell_{\mathrm{kin}, z}^{q}(x) \neq$ $\ell_{\text {can }, z}^{q}(x)$, which implies that the Ji relation does not hold at the density level i.e. that $\left\langle\left\langle J_{\mathrm{Ji}, z}^{q}(x)\right\rangle\right\rangle \neq$ $\frac{1}{2} x\left[H_{q}(x, 0,0)+E_{q}(x, 0,0)\right]$.

- All in all this is a very exciting and open field, both for theory and for experiment. Lattice calculations disagree with each other, and model calculations disagree with each other and with the Lattice results..... a real challenge to theory. On the other hand much extremely interesting information can, in principle, be extracted from experiment, but this requires a significant increase in the precision of the data........ a tough challenge to experiment.

\section{References}

[1] X.S. Chen, X.F. Lu, W.M. Sun, F. Wang, T. Goldman, Phys. Rev. Lett. 100, 232002 (2008), 0806. 3166

[2] E. Leader, C. Lorce, Phys.Rept. (2013), 1309.4235

[3] X.D. Ji, Phys. Rev. D55, 7114 (1997), hep-ph/9609381

[4] M. Diehl, P. Kroll, Eur.Phys.J. C73, 2397 (2013), 1302.4604

[5] J.O. Gonzalez-Hernandez, S. Liuti, G.R. Goldstein, K. Kathuria, Phys.Rev. C88, 065206 (2013), 1206.1876

[6] M. Deka, T. Doi, Y. Yang, B. Chakraborty, S. Dong et al. (2013), 1312.4816

[7] D. Kiptily, M. Polyakov, Eur.Phys.J. C37, 105 (2004), hep-ph/0212372 
[8] M. Penttinen, M.V. Polyakov, A. Shuvaev, M. Strikman, Phys.Lett. B491, 96 (2000), hep-ph/0006321

[9] Y. Hatta, S. Yoshida, JHEP 1210, 080 (2012), 1207.5332

[10] C. Lorcé, B. Pasquini, Phys.Rev. D84, 014015 (2011), 1106.0139

[11] A.W. Thomas, Phys.Rev.Lett. 101, 102003 (2008), 0803.2775

[12] M. Wakamatsu, Eur.Phys.J. A46, 327 (2010)

[13] M. Burkardt, Phys.Rev. D62, 071503 (2000), hep-ph/0005108
[14] J. She, J. Zhu, B.Q. Ma, Phys.Rev. D79, 054008 (2009), 0902. 3718

[15] H. Avakian, A. Efremov, P. Schweitzer, F. Yuan, Phys.Rev. D81, 074035 (2010), 1001.5467

[16] C. Lorcé, B. Pasquini, Phys.Lett. B710, 486 (2012), 1111.6069

[17] M. Burkardt, B.C. Hikmat, Phys. Rev. D79, 071501 (2009), 0812. 1605

[18] P. Hoodbhoy, X.D. Ji, W. Lu, Phys.Rev. D59, 014013 (1999), hep-ph/9804337 